\title{
Pediatric radiation oncology
}

\author{
Arnold C. Paulino
}

Received: 17 February 2013 / Accepted: 20 February 2013 /Published online: 13 March 2013

(C) Springer-Verlag Berlin Heidelberg 2013

Pediatric radiation oncology is a subspecialty that deals with the treatment of children using radiation. While cure is the primary goal in many patients, the avoidance of late effects is equally important as approximately $80 \%$ of patients with childhood tumors are expected to be cured in North America, western Europe, and other industrialized nations. The radiotherapeutic approach to children is quite different in many ways compared to adults. Why is pediatric radiation oncology different from adult radiation oncology?

Pediatric cancer is uncommon. In the USA, there are only 11,000 to 12,000 cases per year, accounting for only $0.7 \%$ of the total cancer cases. While leukemias, lymphomas, and brain tumors comprise about $60 \%$ of all childhood cancer cases; the rest primarily consist of a heterogeneous group of tumors such as neuroblastoma, Wilms' tumor, rhabdomyosarcoma, and various soft tissue and bone sarcomas. Because of their frequency, very few single institution studies with large number of patients have been performed. In North America, several study groups such as the Children's Cancer Group, Pediatric Oncology Group, National Wilms' Tumor Study, and Intergroup Rhabdomyosarcoma Study have pioneered the current backbone of treatment for many of these tumors. Whereas more than $60 \%$ of children are enrolled on protocols, only about $3 \%$ of adults are enrolled in studies.

Training radiation oncology residents to be proficient in the pediatric radiation oncology can be problematic. In one study, only 75 to $80 \%$ of senior residents felt they received adequate training in pediatric radiation oncology during residency. Furthermore, the average resident saw 11 to 20 cases during the entire residency [1]. Because of concerns that some residents may not see enough pediatric cases, the

\footnotetext{
A. C. Paulino $(\square)$

The Methodist Hospital, Houston, TX, USA

e-mail: apaulino@tmhs.org
}

Radiation Oncology Residency Review Committee (RRC) of the Accreditation Council for Graduate Medical Education (ACGME) in the USA has mandated that all residents graduating from an ACGME accredited program see a minimum of 13 pediatric cases during residency. The Radiation Oncology RRC has further stipulated in the Radiation Oncology program requirements that 8 of the required 13 cases be solid tumors. Given the vast heterogeneity of pediatric tumors and their mode of presentation, the resident graduating from an accredited radiation oncology program who is interested in practicing pediatric radiation oncology is likely to need further study either through a fellowship in pediatric radiation oncology or informal training.

While many concepts in pediatric and adult radiation oncology are the same, there is one big difference in the radiotherapeutic management of children. Children are more likely to suffer from radiotherapy late effects compared to adults. The main difference in late toxicity has to do with the developing organs that are being irradiated in children compared to the mature organs in adults [2]. As different organs in the body develop at different times in a child, the time when a particular organ is rapidly developing is the time when the organ is most susceptible to late toxicity. For example, the normal brain of a 3-year old is more susceptible to cognitive deficits than the brain of a 13-year old. Similarly, the breast tissue of a 13-year old is more susceptible to radiation carcinogenesis compared to the same organ in a 3-year old or a 23year old. Knowledge of organ development and radiation tolerance of various developing organs is needed in the radiotherapeutic approach to children. Children are also more likely to develop secondary tumors compared to adults.

Finally, children need support from various fields of medicine that adults may not normally need. The radiation oncology team which includes the physician, therapists, 
nurses, dosimetrists, and physicist need to be aware of issues unique to children. Support personnel such as the anesthesia technologists, social worker, protocol coordinator, child life specialist, psychologist, and recreational therapist are also helpful in the management of children. Physicians such as endocrinologists and anesthesiologists play an active role in pediatric radiation oncology. Finally, the radiotherapy facility should be able to provide anesthesia services including an acute care unit for recovery as well as various types of modern technology (intensity modulated radiotherapy, radiosurgery, proton therapy when available) to treat childhood tumors with the least toxicity.

The state of pediatric radiation oncology in some developing nations lags behind those of the USA and many industrialized nations. One effort in the dissemination of knowledge to radiation oncologists in developing nations is a recent meeting held in Southeast Asia. The symposium, "Pediatric Tumors Revisited," was held on January 27 to 28, 2012 in Manila and featured 14 lectures from five North American pediatric radiation oncologists. In this issue of the journal, five review papers summarize some of the topics featured in this conference. The epidemiology, diagnosis, work-up, and treatment of various tumors are discussed by Dr. Mahajan (lowgrade glioma), Dr. Esiashvili and colleagues (rhabdomyo sarcoma), Dr. Roberts and colleague (non-rhabdomyosarcoma soft tissue sarcoma), and Dr. Chuba (Ewing sarcoma). Finally, I discuss the efforts in the pediatric oncology world to decrease late toxicity of radiotherapy.

\section{References}

1. Gondi V, Bernard JR Jr, Jabbari S, Keam J, de Amorim Bernstein KL et al (2011) Results of the 2005-2008 Association of Residents in Radiation Oncology survey of chief residents in the United States: clinical training and resident working conditions. Int J Radiat Oncol Biol Phys 81(4):1120-1127

2. Paulino AC, Constine LS, Rubin P, Williams JP (2010) Normal tissue development, homeostasis, senescence, and the sensitivity to radiation injury across the age spectrum. Semin Radiat Oncol 20(1):12-20 